\title{
Comparing the effects of general anesthesia and spinal anesthesia on the serum level of blood sugar in patients undergoing cesarean
}

\author{
SAMANEH KOUZEGARAN ${ }^{1}$, HODA SARJUGHI' ${ }^{2}$, AMIR SABER TANHA ${ }^{3, *}$ \\ ${ }^{1}$ Faculty of Medicine, Department of Pediatrics, Birjand University of Medical Sciences, Birjand, Iran \\ ${ }^{2}$ Department of Anesthesia, Birjand University of Medical Sciences, Birjand, Iran \\ ${ }^{3}$ Faculty of Medicine, Department of Anesthesia, Birjand University of Medical Sciences, Birjand, Iran \\ *Corresponding author: Amir Saber Tanha; Faculty of Medicine, Department of Anesthesia, Birjand University of Medical Sciences, 2nd floor, \\ No. 64, Bahonar Sharghi Ave, Pasdaran Ave, Birjand, Iran; Phone: +98 915160 0384; Fax: +98 563243 2778; E-mail: dr.saber@bums.ac.ir
}

\author{
(Received: February 4, 2018; Accepted: March 5, 2018)
}

\begin{abstract}
Objective: This paper aims to study the influence of both general and spinal anesthesia on blood sugar changes in patients undergoing cesarean. Methods: This is a single-blind clinical trial study conducted on 60 patients with classes 1 and 2 anesthesia. The patients were divided by chance into the general anesthesia and spinal anesthesia groups (each contains 30 members). The patients in the spinal group were blocked up to the T4 level and similar methods and medicines were also utilized for those in the general anesthesia group. Results: No significant difference was observed in the blood sugar levels of both groups before and after the operation, but the group, which had utilized the general anesthesia method, had reported with lower levels of blood sugar than the spinal anesthesia group after operation with a significant difference $(P<0.05)$. A difference has been observed in the levels of blood sugar at 2,12 , and $24 \mathrm{~h}$ after the operation in both groups, but it was not a statistically significant difference. Conclusion: However, none of the mentioned methods had any influence on reduction of blood sugar levels during the recovery or the period after it.
\end{abstract}

Keywords: blood, glucose, cesarean, spinal anesthesia, general anesthesia, serum level

\section{Introduction}

Particular metabolic or hormonal changes take place in all types of surgeries basically as a result of sympathy-adrenal stimulation and they have a catabolic nature [1]. Afferent impulses from the sight of stimulation get to hypothalamic and pituitary shaft and result in hormonal changes, such as higher levels of growth hormone and cortisol, stimulation of the efferent flow of sympathy-adrenal, higher levels of catecholamines, aldosterone, and glucagon, and also changes in plasma proteins, sodium retention, potassium loss, and increase in blood sugar [2].

Higher levels of sympathetic activity and noradrenaline result in less insulin secretion. A rise in the level of gluconeogenesis and the less consumption of glucose result in hyperglycemia and there are evidences indicating a resistance toward insulin [3].
Following each type of stress like inducing and retaining anesthesia and the process of surgery, a set of responses known as stress response are induced during which the important organs of the body such as the nervous, endocrine, and immunological systems are affected and biochemical changes take place in the cell. Meanwhile, the endocrine system activates two hypothalamic-pituitary adrenocortical and sympathy-adrenal paths with the final results being the rise or reduction in the levels of some hormones [4]. On one hand, as a result of the influence of various hormones (reduction of insulin and rise of glucagon, catecholamine, cortisol, and growth hormone), blood sugar goes up and this will interrupt blood sugar control during the surgery. On the other hand, various researches have proved the adverse effects of high blood sugar level, such as delayed wound healing and longer period of the patients' hospitalization [5].

This is an open-access article distributed under the terms of the Creative Commons Attribution-NonCommercial 4.0 International License, which permits unrestricted use, distribution, and reproduction in any medium for non-commercial purposes, provided the original author and source are credited, a link to the CC License is provided, and changes - if any - are indicated. 
The majority of the researchers believe that high blood sugar during the surgery in the case of ischemia will result in irrecoverable brain leisure [6]. The studies that have investigated the glucose level of blood during $48 \mathrm{~h}$ after the operation have all pointed to the fact that reduction of stress and anxiety during the operation may prevent high blood sugar and its side effects [7].

It is believed that blocking these stressful reactions through general anesthesia may contribute to controlling these adrenergic reactions. It is also thought that environmental blocking through epidural and spinal in the low level harnesses adrenergic reactions to stress and plays a major role in controlling such changes [8]. Following the earlier researches, the influence of general and spinal anesthesia on reduction of surgery stress and less blood sugar following the operation has been proved; however, the preference of none of these methods has been proved yet [9].

On the other hand, pregnant women during their delivery exhibit increased serum levels of stress hormones (cortisol and epinephrine). Both these hormones contribute to higher serum glucose in the patient [10]. This research seeks a preferable method for controlling the stress caused by the operation and reducing the blood sugar level changes so that in the cases where both the methods are feasible, the preferred method may be utilized to reduce the morbidity effects caused by high blood sugar.

\section{Materials and Methods}

This single-blind random clinical trial study was first verified by the ethics committee of Birjand University of Medical Sciences and gained the permit number IRCT2015030821383N1 from clinical trial registration center. It was conducted on 60 candidates with the physical class of American Society of Anesthesiologists (I \& II) and blood sugar level below 80 who had resorted to Vali-e-Asr Hospital for cesarean. The written consent of all the patients was obtained before beginning the tests. Systematic random method was utilized to divide the patients into two groups (based on the anesthesia method) each comprising 30 people. The information gained through the research was registered in a separate questionnaire for each patient.

Intraoperative monitoring included checking heart pulse and blood pressure. The exclusion criteria included any type of cardiovascular diseases, diabetes, alcoholism, drug consumption or taking drugs, which affect the blood sugar, emergency operations, and any prohibition of general and spinal anesthesia. Some demographic information obtained from the patients including name, surname, age, weight, body mass index, number of pregnancies, history of diabetes in family, cesarean reason, and history of addiction and smoking.
In the next stage, the patient's blood sugar was measured before the operation (anesthesia procedure), after surgical incision, and right after the surgery (when the surgeon was done). Equal amounts of Ringer serum were used for both groups. The medical diet was the same in general anesthesia method, but bupivacaine was applied for spinal anesthesia [11].

For those patients who had undergone spinal anesthesia, the sensory levels after spinal anesthesia and when the senses of the patient were completely restored and the frequency of applying pain reliever were recorded.

After 2, 12, and $24 \mathrm{~h}$ of the operation, the patient's blood sugar level was measured by an expert anesthesiologist through the second finger of the right hand using an Accu-Chek calibrated glucometer with due observation of sampling rules and exclusion of the effective factors, such as triglyceride less than $500 \mathrm{mg} / \mathrm{dl}$ and dehydration hematocrit less than $20 \%$ or more than $65 \%$.

The blood sugar level measured in each stage was subtracted from the level registered in the previous stage. The same procedure was undertaken for all hours and the resulting data were finally analyzed using SPSS. The blood sugar levels of the patient before the operation, during the operation, and at 2, 12, and $24 \mathrm{~h}$ after the operation were measured and subtracted from the preoperation level and the resulting information was fed into SPSS.

Independent $T$-test was used to compare the average blood sugar levels in various stages and coupled $T$-test was utilized to compare the averages before and after the anesthesia. As it is possible that a longer period of fasting may alter the blood sugar of the patients, all the patients were asked to fast at least $8 \mathrm{~h}$ before the operation. For this purpose, the patients were hospitalized 1 day before the operation.

\section{Results}

The groups were similar to one another in terms of gender, age of pregnancy, and type of operation. The results of this study are represented in Tables I-III.

\section{Discussion}

The pain and anxiety of surgery results in higher serum levels of blood sugar through stimulation of sympathetic nerves and secretion of some hormones, such as epinephrine, norepinephrine, and cortisol. Reducing the pain and stress of the surgery through general or spinal anesthesia may prevent sympathetic stimulation and secretion of hormones that increase the blood sugar levels. As a result, no increase in blood sugar levels will take place [12]. 
Kouzegaran et al.

Table I Comparing the demographic variables of patients in both general and spinal anesthesia groups

\begin{tabular}{lccc} 
Group & General anesthesia & Spinal anesthesia & Statistical test \\
Age & $28.77 \pm 4.71$ & $30.46 \pm 5.43$ & $d f=58, t=-2.01, P=0.4$ \\
Weight & $71.88 \pm 12.43$ & $67.33 \pm 7.97$ & $d f=58, t=0.33, P=0.5$ \\
Length of operation & $50.37 \pm 15.05$ & $43.33 \pm 7.71$ & $d f=58, t=1.8, P=0.5$ \\
\hline
\end{tabular}

Table II Comparing the average blood sugar of patients in various stages in both general and spinal anesthesia groups

\begin{tabular}{lccc} 
Group & General anesthesia & Spinal anesthesia & Statistical test \\
Before operation & $86.25 \pm 10.02$ & $92.13 \pm 19.22$ & $d f=40, t=-1.3, P=0.1$ \\
During operation & $83.7 \pm 20.51$ & $95.93 \pm 21.71$ & $d f=40, t=-1.8, P=0.9$ \\
After operation & $93.62 \pm 15.81$ & $110.26 \pm 63.96$ & $d f=40, t=-1.29, P=0.03$ \\
2 h after operation & $88.55 \pm 20.5$ & $96.4 \pm 20.86$ & $d f=40, t=-1.18, P=0.9$ \\
12 h after operation & $104.4 \pm 29.69$ & $102.93 \pm 22.39$ & $d f=40, t=0.16, P=0.1$ \\
24 h after operation & $117 \pm 35.63$ & $115.26 \pm 41.17$ & $d f=40, t=0.16, P=0.7$ \\
\hline
\end{tabular}

Table III Comparing the difference between blood sugar levels during the operation, right after the operation, and at 2, 12 , and $24 \mathrm{~h}$ after the operation with the level recorded before the operation in both groups

\begin{tabular}{lccc} 
Group & General anesthesia & Spinal anesthesia & Statistical test \\
Before operation & $69.6 \pm 12.3$ & $87.4 \pm 22.11$ & $d f=40, t=-1.4, P=0.1$ \\
During operation & $83.2 \pm 18.32$ & $93.98 \pm 22.1$ & $d f=40, t=-1.2, P=0.9$ \\
After operation & $91.60 \pm 14.82$ & $112.30 \pm 62.70$ & $d f=40, t=-1.30, P=0.04$ \\
2 h after operation & $87.30 \pm 19.5$ & $95.3 \pm 22.88$ & $d f=40, t=-1.17, P=0.9$ \\
12 h after operation & $105.6 \pm 30.70$ & $105.90 \pm 21.40$ & $d f=40, t=0.16, P=0.1$ \\
24 h after operation & $120 \pm 34.52$ & $118.30 \pm 40.20$ & $d f=40, t=0.16, P=0.7$ \\
\hline
\end{tabular}

Some researchers have merely focused on studying the changes in blood sugar levels during spinal anesthesia. Based on the results, the type of the medicine used in spinal anesthesia influences the changes in blood sugar. In a study conducted by Fah et al. [13] on patients undergoing cesarean with lidocaine $5 \%$, a significant reduction was observed between the serum level of blood sugar during the operation and that recorded before the surgery. In another study conducted by French et al. [14] on patients undergoing cesarean with bupivacaine $5 \%$, a reduction was observed between the serum level of blood sugar during the operation and that recorded before the surgery. Despite the rise of blood sugar level of the patients during the recovery, it was still less than the level recorded before the operation. According to our research, the serum level of blood sugar level in patients who had undergone spinal anesthesia did not exhibit much difference compared with the preoperation state. However, a significant difference was observed in the blood sugar level during the recovery stage compared with that was recorded during the preoperation stage, but this difference was subsided $2 \mathrm{~h}$ after the surgery. On the other hand and contrary to the previous researches, we studied the serum level of sugar at 12 and $24 \mathrm{~h}$ after the operation. The results indicated further increase in blood sugar during these two periods compared with the levels recorded before the surgery. We may conclude that the spinal anesthesia has a major role in preventing the blood sugar level from going up specially during the operation; however, after the operation and recovery and because of the problems and anxiety of the patient following the side effects of anesthesia, such as nausea and vomiting and shortness of breath, this effect can be subsided [15]. Following the reduction of the side effects of anesthesia, we observe a reduction in blood sugar levels at $2 \mathrm{~h}$ after the operation. After the effects of the anesthesia are subsided and the pain is back, stressful hormones are released and we will observe a rise in the blood sugar level at 12-24 h after the surgery $[16,17]$. 
The effects of general anesthesia on blood sugar level changes have been investigated in some researches. In the study conducted by Chung et al. [18] on 230 patients undergoing eye elective surgery with classes 1 and 2 anesthesia, the serum level of blood sugar exhibited a significant rise after the operation compared with the level recorded before the operation. In another study conducted by Kumar et al. [19] applying for dental surgery using propofol or isoflurane, the average blood sugar of the patients after the operation and during the recovery exhibited a rising pattern in both groups. Contrary to the aforementioned studies, this research found no significant difference in the blood sugar level during, after, and even $2 \mathrm{~h}$ after surgery, but a significant increase was observed at 12 and $24 \mathrm{~h}$ after operation. The differences observed between this research and the studies just mentioned may be due to the difference in the intervening factors, such as the type of operation and physiological changes during the pregnancy [20].

Few studies have compared the effect of general and spinal anesthesia on blood sugar level changes. In a study conducted by Galet et al. [21] of hernia repair surgery under general and spinal anesthesia, the difference between the blood sugar levels at 1 and $6 \mathrm{~h}$ after the operation and the level recorded before the operation was calculated and compared. The results showed a significant reduction in the blood sugar of the patients undergoing spinal anesthesia within $1-6 \mathrm{~h}$ after the operation in comparison with the patients undergoing general anesthesia. The blood sugar of the patients was not measured during the operation. In this research, the difference between the blood sugar levels during, right after, and at 2, 12, and $24 \mathrm{~h}$ after the operation in each group was calculated and compared against the values recorded before the operation [22-24].

Our results indicate greater decreases of blood sugar level in the general anesthesia group than that was observed in the spinal anesthesia group $(P=0.03)$. The studies conducted on patients right after and at 2,12 , and $24 \mathrm{~h}$ after the surgery also showed greater levels of blood sugar increases among those undergoing spinal anesthesia than those undergoing general anesthesia, but these differences were not significant.

This research has not studied the influence of the depth of anesthesia on the blood sugar level of the patient, although the differences in the depth of anesthesia are important factors, which reduce secretion of stress hormones and, as a result, blood sugar. Similar researches that measure the depth of anesthesia and its effects on the blood sugar level are recommended. Furthermore, considering the small sample utilized in this research, similar researches with larger samples are also recommended.

As the results indicate, both the general and spinal anesthesia influence the serum level of the blood sugar in patients undergoing cesarean; however, general anesthesia has a greater effect on increase of blood sugar than spinal anesthesia $(P=0.03)$. None of these methods is effective in reducing the blood sugar level of the patients during the recovery and hours following it.

$$
* * *
$$

Funding sources: Funding was provided by Birjand University of Medical Sciences.

Authors' contribution: SK and HS: study concept and design; acquisition of data, data analysis, and interpretation; and drafting of the manuscript. AST: acquisition of data, data analysis, and interpretation; critical revision of the manuscript; and writing. All authors read and approved the final manuscript.

Conflict of interest: There is no conflict of interest or financial disclosure for this study.

Acknowledgements: The authors would like to thank all patients and hospital staffs to participate in this study.

\section{References}

1. Collins VJ (1993): Principles of Anesthesiology: General and Regional Anesthesia (3rd ed.). Lea and Febiger, Philadelphia, PA

2. Wilmore DW: Alterations in protein, carbohydrate, and fat metabolism in injured and septic patients. J Am Coll Nutr 2, 3-13 (1983)

3. Diltoer M, Camu F: Glucose homeostasis and insulin secretion during isoflurane anesthesia in humans. Anesthesiology 68, 880886 (1988)

4. Vriesendorp TM, Morelis QJ, Devries JH, Legemate DA, Hoekstra JB: Early post-operative glucose levels are an independent risk factor for infection after peripheral vascular surgery. A retrospective study. Eur J Vasc Endovasc Surg 28, 520-525 (2004)

5. Huang YC, Lui PW, Chu CC, Lur JY, Lee TY: Effects of glucosefree maintenance solution on plasma glucose during anesthesia in patients undergoing long neurologic surgery. Zhonghua Yi Xue Za Zhi (Taipei) 63, 467-474 (2000)

6. Zahedi H, Akhyani V, Hussainkhan Z, Younesian M: Evaluation of FBS changes before, during and after elective eye surgery under general anesthesia, with glucometer and lab tests. TUMJ 1385, 30-35 (2007)

7. Halter JB, Pflug AE: Effect of sympathetic blockade by spinal anesthesia on pancreatic islet function in man. Am J Physiol 239, E150-E155 (1980)

8. Veering BT, Burm AG, van Kleef JW, Hennis PJ, Spierdijk J: Spinal anesthesia with glucose-free bupivacaine: Effects of age on neural blockade and pharmacokinetics. Anesth Analg 66, 965-970 (1987)

9. Cheek TG, Gutsche BB, Gaiser RR (1999): Obstetric anesthesia: Principles and practice. In: The Pain of Childbirth and Its Effect on the Mother and Fetus, ed Chestnut DH, Mosby, St. Louis, pp. 386-408

10. Roizen MF, Michael F, Lee AF (2010): Anesthetic implication of concurrent disease. In: Miller's Anesthesia, ed Miller RD, Elsevier, Philadelphia, p. 3576

11. Arthur GR, Scott DH, Boyes RN, Scott DB: Pharmacokinetic and clinical pharmacological studies with mepivacaine and prilocaine. Br J Anaesth 51, 481-485 (1979)

12. Bannister J, McClure JH, Wildsmith JA: Effect of glucose concentration on the intrathecal spread of $0.5 \%$ bupivacaine. Br J Anaesth 64, 232-234 (1990)

13. Fah A, Sutton J, Cohen V, Dowling K, Cyna AM: A comparison of epidural and cerebrospinal fluid glucose in parturients at term: An observational study. Int J Obstet Anesth 21, 242-244 (2012) 
14. French GW, White JB, Howell SJ, Popat M: Comparison of pentastarch and Hartmann's solution for volume preloading in spinal anaesthesia for elective caesarean section. Br J Anaesth 83, 475-477 (1999)

15. Hahn RG, Resby M: Volume kinetics of Ringer's solution and dextran 3\% during induction of spinal anaesthesia for caesarean section. Can J Anaesth 45, 443-451 (1998)

16. Burm AG, van Kleef JW, Gladines MP, van Duinen M, Spierdijk J: Spinal anesthesia with hyperbaric lidocaine and bupivacaine: Effects of epinephrine on the plasma concentration profiles. Anesth Analg 66, 1104-1108 (1987)

17. Cousins MJ, Bromage PR (1988): Epidural neural blockade. In: Neural Blockade in Clinical Anesthesia and Management of Pain, eds Cousins MJ, Bridenbaugh PO (2nd ed.), JB Lippincott, Philadelphia, pp. 323-371

18. Chung CJ, Bae SH, Chae KY, Chin YJ: Spinal anaesthesia with $0.25 \%$ hyperbaric bupivacaine for caesarean section: Effects of volume. Br J Anaesth 77, 145-149 (1996)
19. Kumar A, Bala I, Bhukal I, Singh H: Spinal anaesthesia with lidocaine 2\% for caesarean section. Can J Anaesth 39, 915-919 (1992)

20. Hirabayashi Y, Shimizu R, Matsuda I, Inoue S: Effect of extradural compliance and resistance on spread of extradural analgesia. Br J Anaesth 65, 508-513 (1990)

21. Galet A, Fleyfel M, Beague D, Vansteenberghe F, Krivosic-Horber R: Accidental spinal anesthesia in obstetrics. Limits of epidural testdose. Ann Fr Anesth Reanim 11, 377-380 (1992)

22. Greene NM: Uptake and elimination of local anesthetics during spinal anesthesia. Anesth Analg 62, 1013-1024 (1983)

23. Kozody R, Swartz J, Palahniuk RJ, Biehl DR, Wade JG: Spinal cord blood flow following sub-arachnoid lidocaine. Can Anaesth Soc J $32,472-478$ (1985)

24. Langerman L, Grant GJ, Zakowski M, Ramanathan S, Turndorf H: Prolongation of spinal anesthesia. Differential action of a lipid drug carrier on tetracaine, lidocaine, and procaine. Anesthesiology 77, 475-481 (1992) 